\title{
Gradiente de Retardo Temporal y Reflexiones en Superficies Corrugadas
}

\author{
Time-delay Gradient and Reflections on Corrugated Surfaces
}

\author{
Georgina Lizaso $^{\# 1}$, Nicolás Casais Dassie ${ }^{\# 2}$, Damián Andrés Fernández ${ }^{\# 3}$, Jorge Petrosino ${ }^{\# 4}$ \\ ${ }^{*}$ Instituto de Producción, Economía y Trabajo. Departamento de Humanidades y Artes. Universidad Nacional de Lanús \\ 29 de septiembre 3901, Remedios de Escalada, Buenos Aires, Argentina

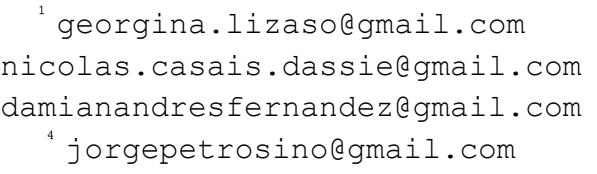 \\ Recibido: 07/12/18; Aceptado: 06/03/19
}

\begin{abstract}
Acoustic wave reflections from corrugated surfaces are analyzed. For this work, a proposal of Yi-Fan Zhu on a relatively simple model of metasurface is taken as a basis. Each groove of a corrugated surface can be considered analogous to a linear array, while the depth of its grooves is related to the phase of each source. Zhu's original proposal takes the phase gradient of the arrangement as a design parameter. In this paper we analyze the advantages of surface design from the time-delay gradient. It also presents the possibility of impose brief delays in the whole pattern of reflections independently of the general design process.
\end{abstract}

Keywords: dispersionless reflection; acoustic metasurfaces; reflection control.

Resumen- Se analizan las reflexiones de ondas acústicas en superficies corrugadas. Para este trabajo se toma como base una propuesta de Yi-Fan Zhu sobre un modelo relativamente sencillo de metasuperficie. Cada ranura de una superficie corrugada puede considerarse análoga a un arreglo lineal de fuentes, mientras que la profundidad de sus hendiduras se relaciona con la fase de cada fuente. La propuesta original de Zhu toma como parámetro de diseño al gradiente de fase del arreglo. En este trabajo se analizan las ventajas del diseño de superficies a partir del gradiente de retardos temporales. Se presenta además la posibilidad de incorporar breves retardos en todo el patrón de reflexiones de modo independiente al proceso general de diseño.

Palabras clave: reflexión sin dispersión metasuperficies acústicas; control de reflexión.

\section{INTRODUCCIÓN}

En los últimos años se verifica un creciente interés en el estudio de estructuras artificiales con detalles espaciales menores a la longitud de onda (subwavelength) que podrían dar lugar a características de comportamiento difícilmente alcanzables con materiales convencionales. Estas estructuras se conocen como metamateriales y la posibilidad de aplicaciones en acústica se mantiene en crecimiento [1]. Un metamaterial es una estructura artificial obtenida por agregación de uno o varios materiales con un diseño particular en relación con su geometría, orientación y organización interna que da lugar a propiedades que no se presentan en materiales naturales. Los componentes de los metamateriales usualmente están organizados en patrones a escalas menores que la longitud de onda de los fenómenos sobre los que influyen. Cuando el diseño de un metamaterial se refiere a un desarrollo en dos dimensiones suele denominarse metasuperficie.

El presente trabajo analiza un tipo particular de estructuras que consideramos interesante como puerta de ingreso al tema de metamateriales acústicos por la potencial simplicidad que ofrece para relacionar los conceptos clásicos con las nuevas estructuras. Tomamos como referencia los trabajos de Zhu y colaboradores [2], [3] en los que se analizan las condiciones que debe cumplir una superficie corrugada para lograr control de reflexión sin dispersión. La propuesta de diseño de Zhu toma como punto de partida el gradiente de fase deseado, lo que obliga a presuponer una frecuencia de referencia para el cálculo aun cuando el resultado del diseño resulte independiente de la frecuencia dentro del rango de validez, sin embargo es posible obtener ecuaciones más sencillas de aplicar si se considera el gradiente deseado de retardos temporales en lugar del gradiente de fase.

Se presentan simulaciones utilizando Comsol Multiphysics y el método pseudoespectral del espacio k [4], [5] con base en las características reportadas por Zhu, su ajuste con los presupuestos teóricos que sustentan su comportamiento así como los criterios de diseño que podrían aplicarse para obtener metamateriales con un comportamiento de reflexión deseado.

\section{A. Frentes de onda emitidos por un arreglo lineal}

Zhu propone partir de la conocida teoría que describe el comportamiento de un arreglo lineal de $\mathrm{n}$ elementos separados por una distancia $\Delta x$. Un arreglo con $n$ fuentes en fase emitirán un frente de ondas que resultará cilíndrico para frecuencias dentro de determinada banda. La dirección de propagación será perpendicular al arreglo (esto es, un ángulo de emisión igual a $0^{\circ}$ ). La frecuencia inferior de la banda estará relacionada con la longitud total del arreglo 
$\mathrm{L}=\mathrm{n} . \Delta \mathrm{x}$ y la frecuencia superior con la distancia $\Delta \mathrm{x}$ entre fuentes.

Una diferencia de fase constante entre fuentes adyacentes dará lugar a un frente de ondas con un ángulo de emisión $\theta$, que depende de la diferencia de recorrido $(\Delta \mathrm{r})$, y de la distancia entre fuentes $(\Delta \mathrm{x})$ como se muestra en la Fig. 1.

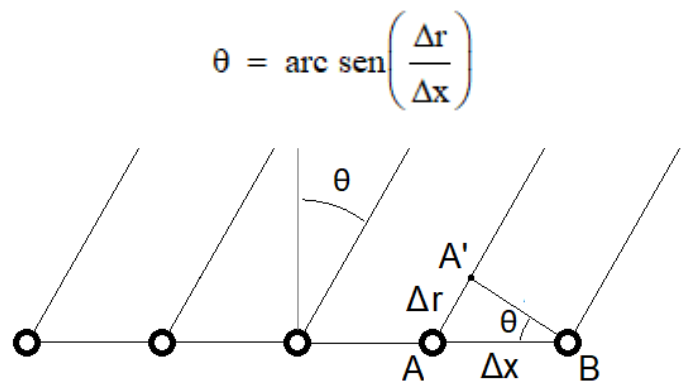

Fig. 1. Un arreglo lineal con diferencia de fase constante entre fuentes emitirá un frente de ondas con un ángulo $\theta$.

Para que los puntos A’ y B de la Fig. 1 estén en fase, es necesario que la fase del elemento A esté adelantada respecto de $\mathrm{B}$ por un valor $\Delta \phi$ que cumpla con la relación

$$
\frac{\Delta \phi}{2 \pi}=\frac{\Delta \mathrm{r}}{\lambda}
$$

Combinando (1) y (2) se obtiene

$$
\theta=\operatorname{arcsen}\left(\frac{\lambda}{2 \pi} \cdot \frac{\Delta \phi}{\Delta x}\right)
$$

Para el caso de considerar un arreglo continuo de fuentes $\Delta \phi / \Delta \mathrm{x}$ se transforma en $\mathrm{d} \phi / \mathrm{dx}$ que es el gradiente de fase del arreglo. La Fig. 2 muestra la propagación del frente de ondas emitido por un arreglo lineal de 12 elementos con gradiente de fase distinto de cero.

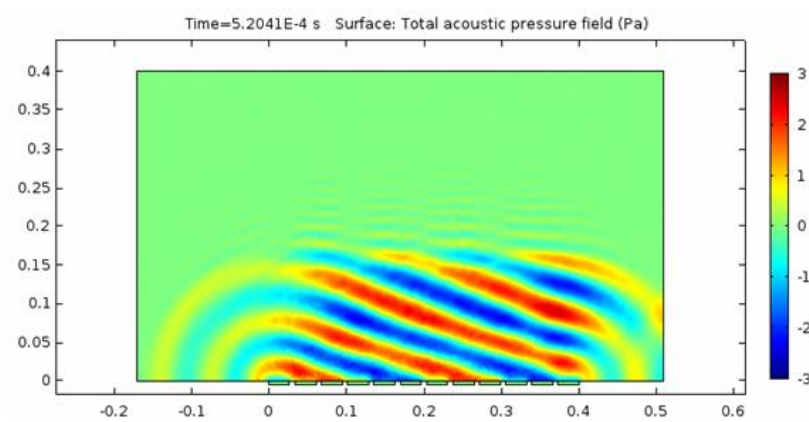

Fig. 2. Frente de onda emitido por un arreglo con gradiente de fase

La Ec. (3) permite analizar las condiciones necesarias para que el ángulo de emisión $\theta$ sea no dispersivo (esto es, que resulte independiente de la frecuencia). El argumento del arco seno depende en forma explícita de la longitud de onda, de modo que para que $\theta$ sea no dispersivo es necesario que el gradiente de fase $d \phi / d x$ sea inversamente proporcional a $\lambda$.

\section{B. Frentes de onda reflejados por un material corrugado}

La Fig. 3 muestra una superficie corrugada formada por un conjunto de hendiduras rectangulares de diferentes profundidades.

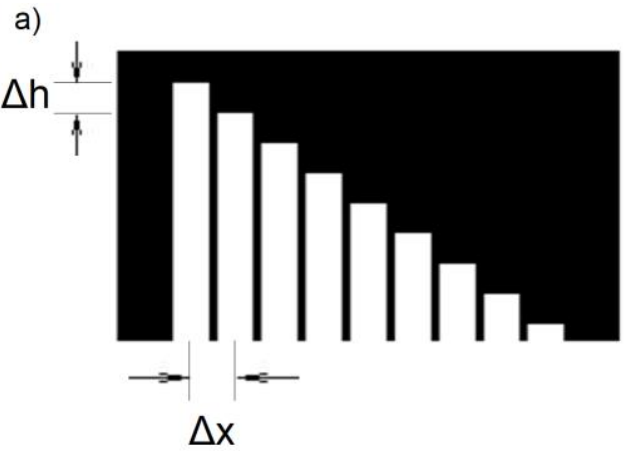

b)

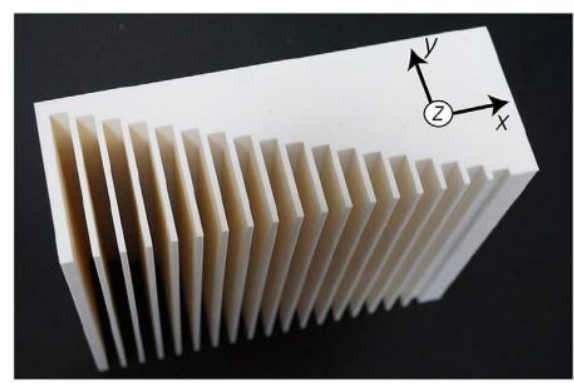

Fig. 3. Superficie corrugada. (a) esquema de la sección, (b) fotografía mostrando la metasuperficie del artículo citado (Zhu, 2015).

Una onda plana que incida en la dirección del eje $y$ (indicado en la Fig. 3b) enfrentará cada extremo abierto de las hendiduras en fase, recorrerá su longitud y regresará para ser emitido por el extremo abierto con su fase cambiada.

El cambio provocado en la fase se debe a una diferencia de recorrido, lo que dará lugar a un gradiente de fase proporcional a $f$ (inversamente proporcional a $\lambda$ ), cumpliendo la condición indicada por Zhu para asegurar reflexión sin dispersión.

La frecuencia inferior del rango estará relacionada con la longitud total del arreglo (en la dirección $x$ de la Fig. 3b) y $\mathrm{su}$ frecuencia superior con la separación entre ranuras $(\Delta \mathrm{x})$.

Dada una ranura de profundidad $\Delta \mathrm{h}$, el tiempo necesario para recorrerla con velocidad de propagación c será

$$
\Delta \mathrm{t}=\frac{2 \cdot \Delta \mathrm{h}}{\mathrm{c}}
$$

Pero además $\Delta \mathrm{t} / \mathrm{T}=\mathrm{f} . \Delta \mathrm{t}=\Delta \phi / 2 \pi$, por lo tanto

$$
\Delta \phi=2 \pi \mathrm{f} \cdot \Delta \mathrm{t}=\frac{2 \pi \mathrm{f}}{\mathrm{c}} \cdot 2 \Delta \mathrm{h}=\frac{2 \pi}{\lambda} \cdot 2 \Delta \mathrm{h}
$$

Reemplazando la ecuación (5) en la Ec. (3), se cancela el factor $\lambda / 2 \pi$

$$
\theta=\operatorname{arc} \operatorname{sen}\left(2 \cdot \frac{\Delta \mathrm{h}}{\Delta \mathrm{x}}\right)
$$

La Ec. (6) relaciona el gradiente de profundidad de las hendiduras $\Delta \mathrm{h} / \Delta \mathrm{x}$ con el ángulo de reflexión sin dispersión.

Por su parte $\Delta \mathrm{h} / \Delta \mathrm{x}$ será la tangente del ángulo de inclinación del plano interno de las hendiduras. Un plano tangente a sus extremos internos inclinado un ángulo $\alpha$ provocará un ángulo de emisión

$$
\theta=\operatorname{arc} \operatorname{sen}(2 \cdot \tan (\alpha))
$$


La Fig. 4 muestra el frente de ondas generado cuando en la Ec. (7) se utiliza $\alpha=25.2^{\circ}$.

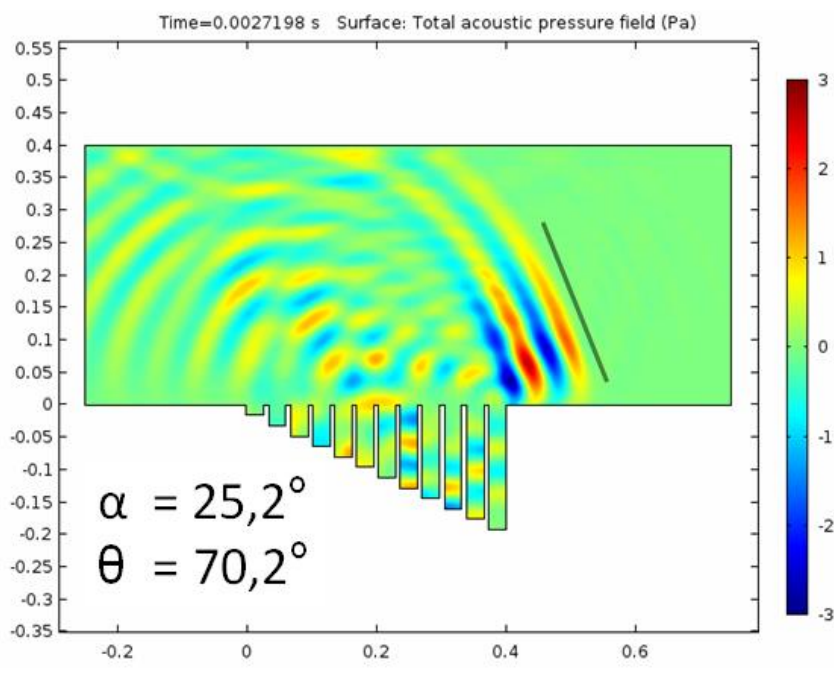

Fig. 4. Reflexión de un frente de onda por la superficie corrugada con ángulo de perfil interno es de $25,2^{\circ}$.

Si se reemplaza la superficie corrugada por un plano con inclinación $\alpha$, el ángulo de emisión será $\theta=2 \alpha$. Para ángulos pequeños la emisión del frente corrugado resulta semejante al generado por la reflexión ante un plano inclinado, pero cambia para ángulos mayores. En la Fig. 5, se muestra la propagación del frente de ondas de una superficie plana con inclinación $\alpha=25.2^{\circ}$.

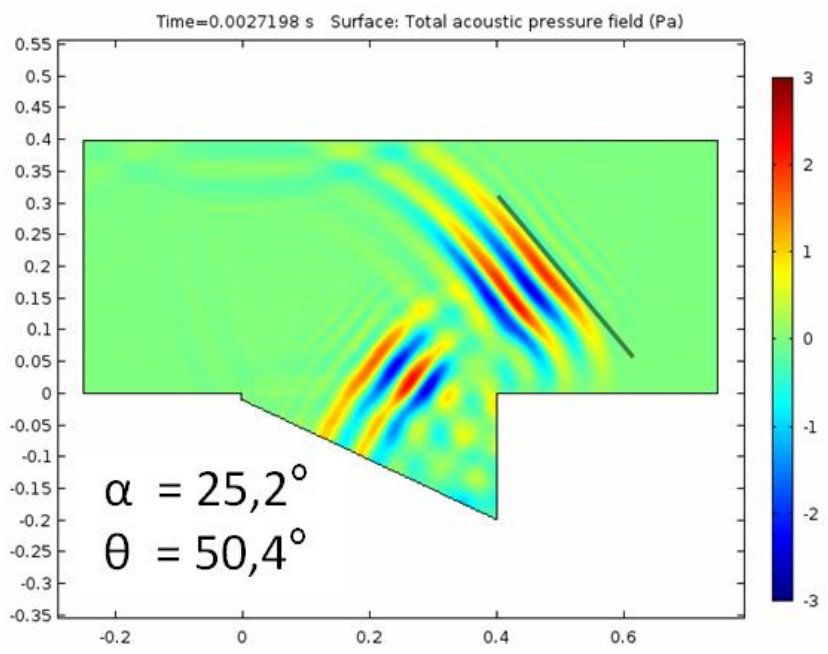

Fig. 5. Reflexión de un frente de onda por la superficie plana con ángulo de inclinación de $25,2^{\circ}$.

\section{Diseño DE PERFILES A PARTIR DEL GRADIENTE DE FASE}

Con las ecuaciones anteriores es posible diseñar un perfil que genere reflexiones no dispersivas con un ángulo $\theta$ deseado. En los planteos anteriores se ha supuesto que el gradiente $\Delta \mathrm{h} / \Delta \mathrm{x}$ es constante, por tratarse del caso más sencillo.

En forma general, la condición de no dispersión podría establecerse reescribiendo la Ec. (5) para un caso general en el cual el perfil de hendiduras $\Delta \mathrm{h}$ pueda ser dependiente de $x$.

$$
\Delta \phi=\frac{2 \pi}{\lambda} \cdot 2 \cdot \Delta \mathrm{h}(\mathrm{x})
$$

Generalizando para el caso de un arreglo lineal continuo puede escribirse

$$
\frac{\mathrm{d} \phi}{\mathrm{dx}}=\frac{2 \pi}{\lambda} \cdot 2 \cdot \frac{\mathrm{dh}}{\mathrm{dx}}
$$

Reorganizando los términos de la ecuación e integrando, se obtiene la Ec. 10, que permite diseñar un perfil $\mathrm{h}(\mathrm{x}) \mathrm{a}$ partir de un gradiente de fases deseado.

$$
\mathrm{h}(\mathrm{x})=\frac{\lambda}{4 \pi} \cdot \int \frac{\mathrm{d} \phi}{\mathrm{dx}} \mathrm{dx}
$$

La ecuación (10) expresa en líneas generales la propuesta de Zhu para obtener perfiles complejos. El proceso requiere tomar una frecuencia f0 como referencia para determinar en ese caso el perfil de fases y el valor de $\lambda$, aun cuando posteriormente el perfil obtenido resulte no dispersivo.

\section{DISEÑO DE PERFILES A PARTIR DEL GRADIENTE DE RETARDOS TEMPORALES}

Existe una alternativa que no requiere tomar como referencia una frecuencia de base para el diseño. Consiste en relacionar el gradiente de profundidad necesario $\mathrm{dh} / \mathrm{dx}$ con el gradiente de retardos temporales deseado $\mathrm{d} \tau / \mathrm{dx}$.

$$
\begin{gathered}
\Delta \phi=2 \pi \mathrm{f} \cdot \Delta \tau=\frac{2 \pi}{\lambda} \cdot \mathrm{c} \cdot \Delta \tau \\
\frac{\mathrm{d} \phi}{\mathrm{dx}}=\frac{2 \pi}{\lambda} \cdot \mathrm{c} \cdot \frac{\mathrm{d} \tau}{\mathrm{dx}}
\end{gathered}
$$

Reemplazando el gradiente de fase de la ecuación (12) en la ecuación (10) se obtiene

$$
h(x)=\frac{c}{2} \cdot \int \frac{d \tau}{d x} d x=\frac{c}{2} \cdot \tau(x)+C
$$

La Ec. (13) permite obtener el perfil de profundidad de hendiduras a partir de la función deseada de retardos en cada elemento del arreglo $\tau(\mathrm{x})$, sin necesidad de suponer una frecuencia de referencia como en la propuesta original de Zhu. La constante de integración $\mathrm{C}$ puede tener cualquier valor arbitrario. En la práctica esto se corresponde con un desplazamiento paralelo al plano horizontal de todo el perfil $\mathrm{h}(\mathrm{x})$ y resulta en un retardo temporal constante para todos los elementos del arreglo. Variando esta constante de integración $\mathrm{C}$ puede conseguirse una reflexión con un retardo respecto del momento en que el frente de onda impacta en la superficie. Este retardo puede controlarse en forma independiente del ángulo de reflexión elegido previamente. 


\section{A. Ejemplo de diseño de reflexión plana}

Con el fin de ejemplificar la aplicación de la Ec. (13), se proponen los siguientes pasos de diseño del perfil para lograr un ángulo de reflexión $\theta=30^{\circ}$. En este caso la diferencia de recorrido entre elementos extremos del arreglo que será $\mathrm{D}=\mathrm{L} \cdot \operatorname{sen}\left(30^{\circ}\right)$

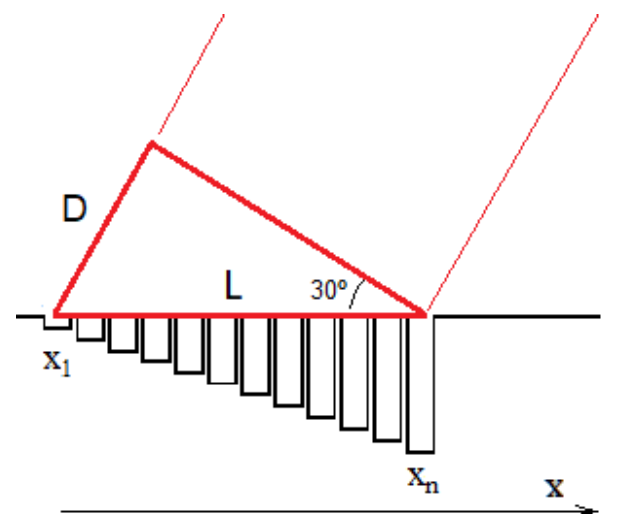

Fig. 6. Perfil del reflector. L es longitud total y D diferencia de recorrido.

Considerando una variable continua $x$ cuyo origen coincida con la primera ranura, a medida que $x$ crece debe incrementarse el retardo de cada reflexión para lograr emitir un frente de ondas con el ángulo indicado en la Fig. 6. Para $\mathrm{x}=\mathrm{L}$ (longitud total del arreglo) el retardo necesario es D/c. El retardo temporal en función de $x$ resulta

$$
\tau(\mathrm{x})=\frac{\mathrm{D}}{\mathrm{c}} \cdot \frac{\mathrm{x}}{\mathrm{L}}=\frac{1}{\mathrm{c}} \cdot \operatorname{sen}\left(30^{\circ}\right) \cdot \mathrm{x}
$$

El perfil h(x) determinado por la ecuación (13) es

$$
\mathrm{h}(\mathrm{x})=\frac{\mathrm{c}}{2} \cdot \tau(\mathrm{x})+\mathrm{C}=\frac{\operatorname{sen}\left(30^{\circ}\right)}{2} \mathrm{x}+\mathrm{C}
$$

Para distintos valores de la constante de integración $\mathrm{C}$ se obtienen diferentes retardos manteniendo el mismo ángulo de reflexión. La Fig. 7 muestra el frente de ondas reflejado luego de $2.6 \mathrm{~ms}$ del inicio de la simulación utilizando $\mathrm{C}=0$. $\mathrm{Al}$ comparar con la simulación que utiliza $\mathrm{C}=0.16 \mathrm{~m}$ (Fig. 8) para el mismo tiempo transcurrido puede notarse que la reflexión se emite con un retardo temporal.

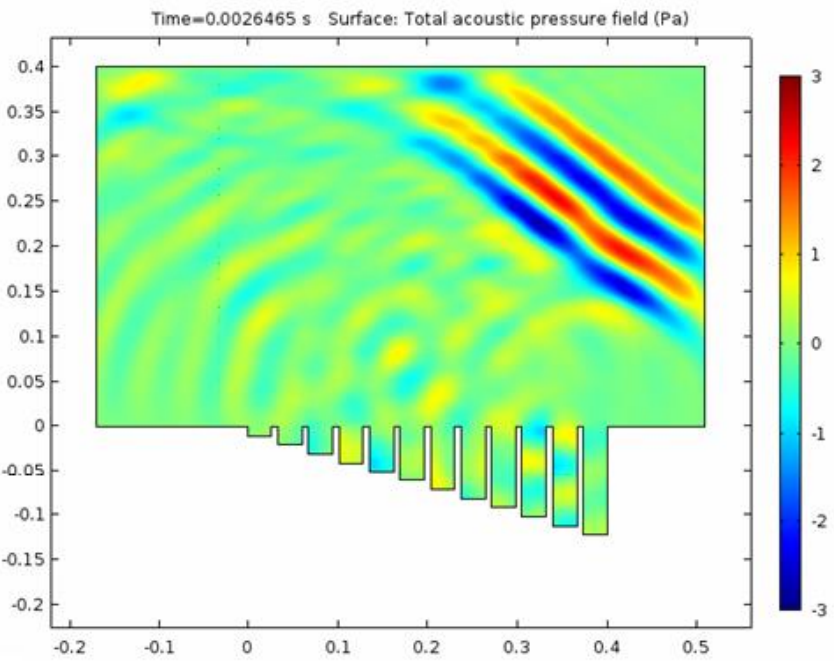

Fig. 7. Reflexión del perfil h(x) para constante de integración C=0.

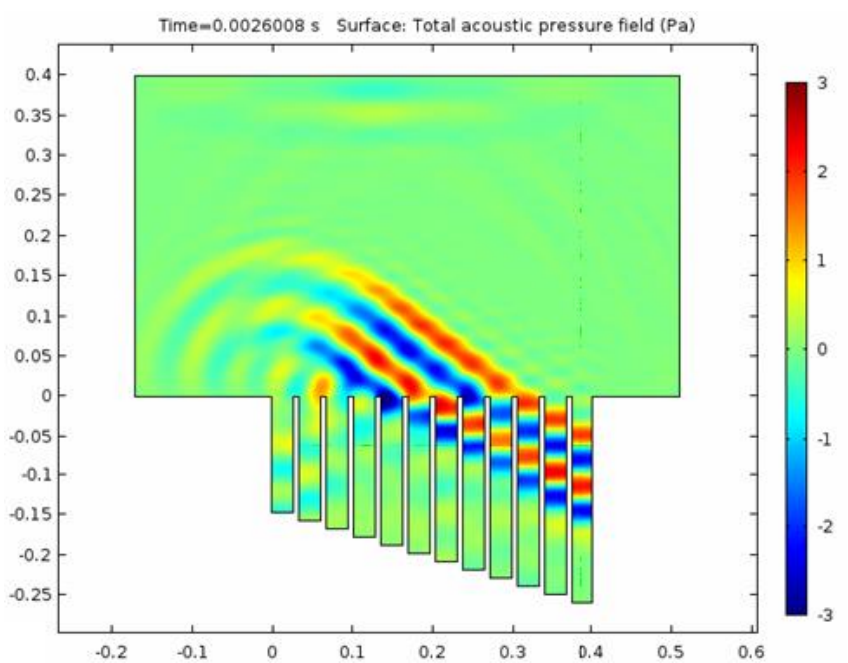

Fig. 8. Reflexión del perfil $\mathrm{h}(\mathrm{x})$ para constante de integración $\mathrm{C}=0.16 \mathrm{~m}$ incorporando un retardo cercano a un milisegundo en la reflexión.

\section{B. Ejemplo de diseño de reflexión compleja}

A partir de la Ec. (13) es posible diseñar un perfil $\mathrm{h}(\mathrm{x})$ conociendo los retardos temporales necesarios para cada elemento del arreglo.

Para diseñar un reflector sin dispersión que concentre un frente de onda plana en un punto determinado se requiere calcular la función de retardos temporales $\tau(\mathrm{x})$ necesaria a partir de las distancias entre el foco y cada ranura (Fig. 9)

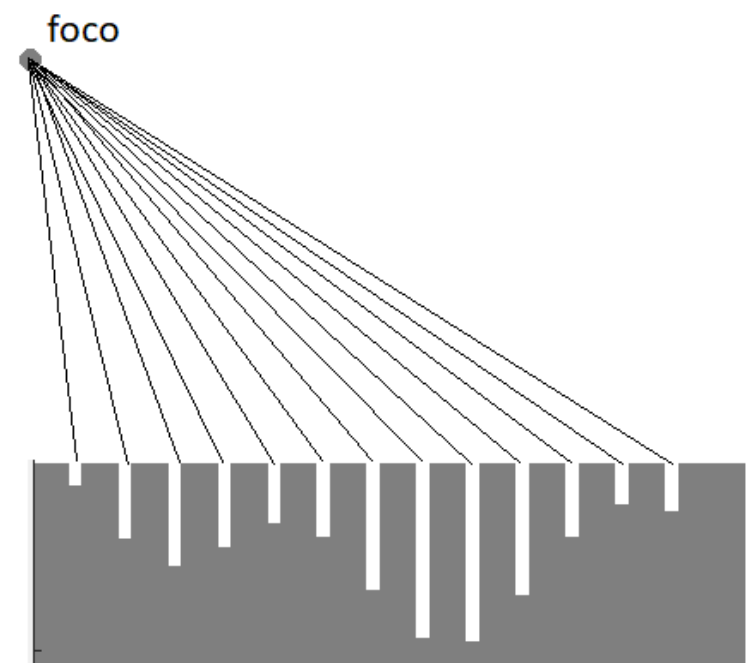

Fig. 9. Distancias entre el foco y cada elemento.

Si el foco se encuentra en $[\mathrm{x} 0, \mathrm{y} 0]$ la función $\tau(\mathrm{x})$ será

$$
\tau(\mathrm{x})=\frac{1}{\mathrm{c}} \cdot \sqrt{(\mathrm{x}-\mathrm{x} 0)^{2}+\mathrm{y} 0^{2}}
$$

A partir de esta expresión es posible obtener

$$
h(x)=\frac{1}{2} \cdot \sqrt{(x-x 0)^{2}+y 0^{2}}+C
$$


Un perfil que responda a la ecuación (17) provocará que una onda plana incidente en forma perpendicular al plano se refleje concentrándose en el punto elegido como foco. La Fig. 10 muestra un instante de la simulación realizada. Es importante notar que si bien el efecto resultante es semejante al que produciría un perfil parabólico el fenómeno involucrado es diferente. La función que define el perfil con hendiduras no es una función proporcional a $x$ al cuadrado sino a la raíz cuadrada de $x$ al cuadrado.

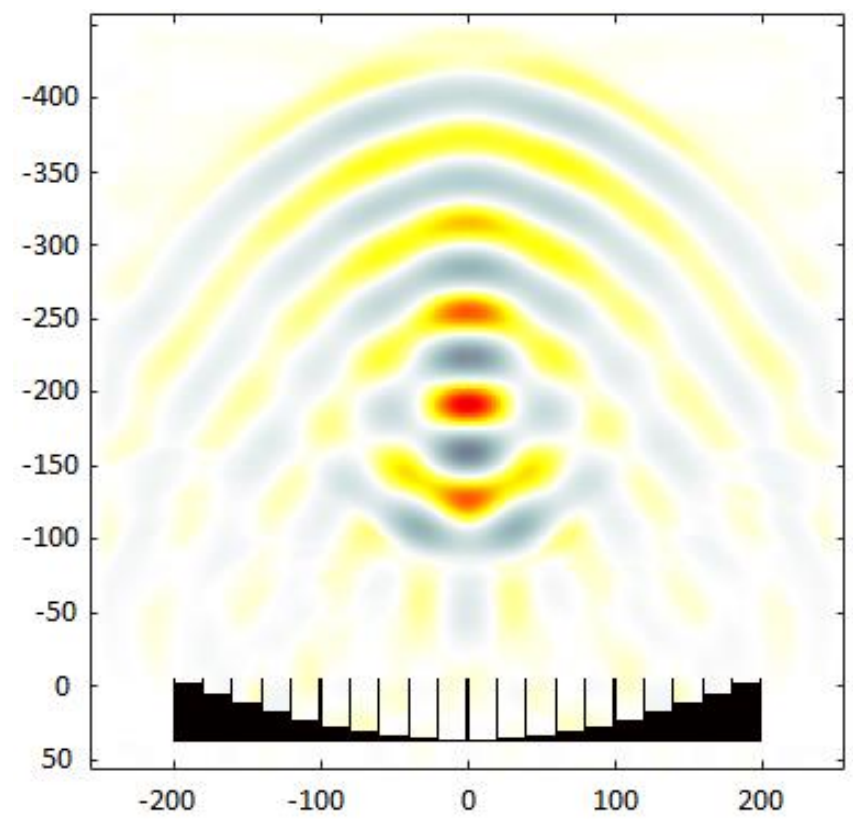

Fig. 10. Simulación de ondas concentrándose en el foco $[0 ; 0.2 \mathrm{~m}]$.

\section{CONCLUSIONES Y TRABAJO FUTURO}

La propuesta de Zhu y colaboradores permite diseñar perfiles corrugados para lograr reflexión sin dispersión de modo controlado dentro de un rango de frecuencias a partir de conocer su gradiente de fase. Nuestra propuesta de reemplazar el gradiente de fase por un gradiente de retardos temporales evita la elección de una frecuencia de referencia arbitraria para el diseño. Por otra parte, permite también que los retardos temporales en cada elemento del arreglo puedan ser determinados mediante cálculo, medición o incluso por simulación, ubicando una fuente en el lugar en el que se desea obtener una concentración de las reflexiones y colocando sensores en el extremo abierto de las hendiduras. Los registros de los sensores resultan ser un muestreo espacial de $\tau(\mathrm{x})$.

Hemos realizado algunas pruebas preliminares para analizar la posibilidad de obtener dos reflexiones de ondas planas o incluso dos puntos focales en forma simultánea con un solo perfil. Sin embargo, es necesario un trabajo más cuidadoso antes de poder presentar estos resultados.

Por otra parte, estamos realizando los modelos físicos de los perfiles mediante impresión 3D con la colaboración de docentes y estudiantes de la Licenciatura en Diseño Industrial de la Universidad Nacional de Lanús con el fin de complementar el presente trabajo con mediciones experimentales (Fig.11, Fig. 12)

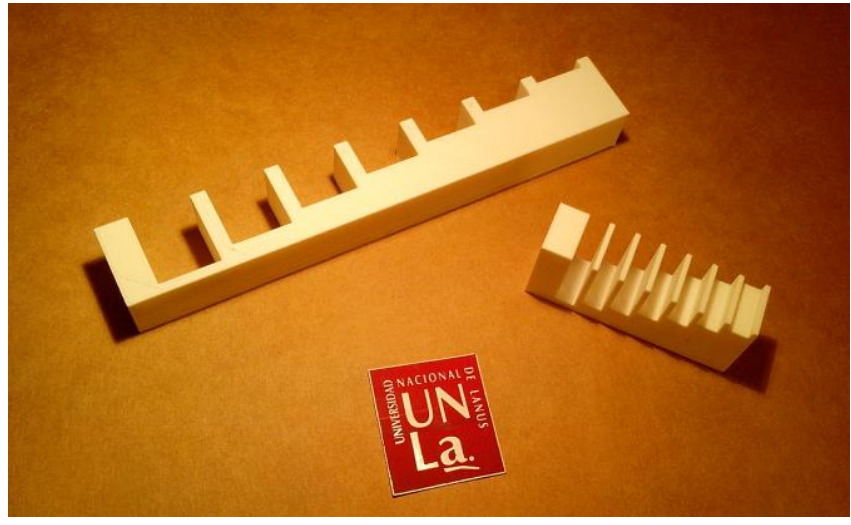

Fig. 11. Impresión 3D de perfiles corrugados.

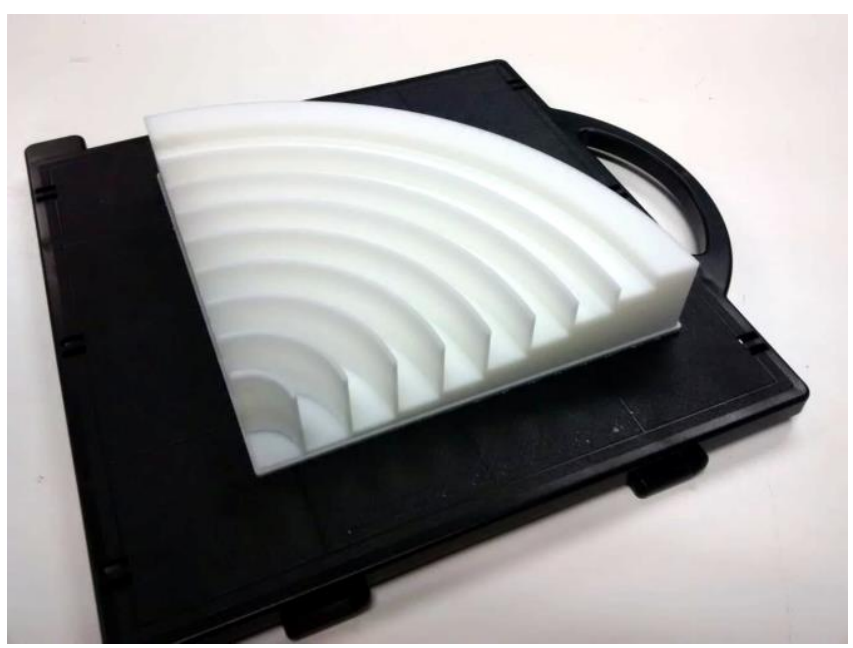

Fig. 12. Impresión 3D del perfil simulado en la Fig. 10.

\section{AGRADECIMIENTOS}

Agradecemos la buena voluntad y el empeño puesto por parte de Andrés Ruscitti, Guillermo Andrade y los estudiantes Lucas Bujan y Sabrina Accotto que están encargándose de modelar e imprimir las primeras pruebas de perfiles dentados planos y circulares para las futuras mediciones.

El presente trabajo es parte de un proyecto de investigación sobre propagación de ondas acústicas que estamos desarrollando en la Universidad Nacional de Lanús conjuntamente con Andrés Bonino Reta, Ianina Canalis, Ian Kuri y Lucas Landini.

\section{REFERENCIAS}

[1] G. Ma, y P. Sheng, "Acoustic metamaterials: From local resonances to broad horizons." Science advances, 2(2), e1501595, 2016

[2] Y. F. Zhu, X. Y. Zou, R. Q. Li, X. Jiang, J. Tu, B. Liang y J. C. Cheng, "Dispersionless manipulation of reflected acoustic wavefront by subwavelength corrugated surface", Scientific reports, 5, 10966, 2015

[3] Y. F. Zhu, X. Y. Zou, B. Liangy J. Cheng, "Acoustic one-way open tunnel by using metasurface", Applied Physics Letters, 107(11), 113501,2015

[4] M. Tabei, T. D. Mast y R. C. Waag, "A k-space method for coupled first-order acoustic propagation equations", The Journal of the Acoustical Society of America, 111(1), pg. 53-63, 2002

[5] B. E. Treeby, y B. T. Cox, "k-Wave: MATLAB toolbox for the simulation and reconstruction of photoacoustic wave fields", Journal of biomedical optics, 15(2), 021314, 2010 